\title{
Numerical modelling of nonlinear electromechanical coupling of an atomic force microscope with finite element method
}

\author{
J. Freitag and W. Mathis \\ Institut für Theoretische Elektrotechnik, Appelstraße 9A, 30167 Hannover, Germany
}

\begin{abstract}
In this contribution, an atomic force microscope is modelled and in this context, a non-linear coupled 3-Dboundary value problem is solved numerically using the finite element method. The coupling of this system is done by using the Maxwell stress tensor. In general, an iterative weak coupling is used, where the two physical problems are solved separately. However, this method does not necessarily guarantee convergence of the nonlinear computation. Hence, this contribution shows the possibility of solving the multiphysical problem by a strong coupling, which is also referred to as monolithic approach. The electrostatic field and the mechanical displacements are calculated simultaneously by solving only one system of equation. Since the Maxwell stress tensor depends nonlinearly on the potential, the solution is solved iteratively by the Newton method.
\end{abstract}

\section{Motivation}

Due to the tremendous development of science in the field of nanotechnology over the last years, microsystems can increasingly be used on many levels. Compared to macrosystems, the advantage lies in cost savings (less consumption of materials, parallel production) and efficiency (low energy and power requirements allow autonomous systems). Applications for these microsystems are primarily medical and security systems, life sciences and logistics. This process makes the use of high resolution instrumentation and its optimisation indispensable to produce these systems. In this context, scanning probe microscopy (SPM) has been developed to be the most appropriate procedure. Here a sample is scanned pointwise using a probe. The resulting measurements are assembled and used to finally produce an image of the object to be measured in terms of the considered interactions. Since atomically small structures are measured, the process is highly error liable due to various interferences, which makes the interpretation of the results difficult. The

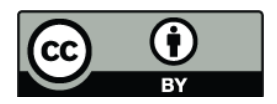

Correspondence to: J. Freitag

(freitag@tet.uni-hannover.de) aim of this work is to simulate the measurement process to reflect the impact of such measurement errors and eliminate them from the measurements data.

\section{Scanning Probe Microscopy}

The existing types of SPMs differ in the kind of interaction (electric, magnetic, etc.) between the tip and the sample. This contribution is considered with the atomic force microscope (AFM). The AFM was developed in 1986 and measures atomic forces on a nanometer scale. With a resolution from 0.1 to $10 \mathrm{~nm}$ and a scanning velocity from 0.5 to 10 lines per second, it could last $20 \mathrm{~min}$ until a digital picture emerges. The AFM consists of a cantilever spring with a sharp tip on the end, which is ideally conical and has a diameter of one atom (Fig. 1). During the process of measuring, the tip navigates above the sample, leading to the potential difference. The cantilever deflects due to the adhesive interactions. This change on the position of the cantilever is tracked by a laserbeam, which points at a position sensitive photodetektor. This signal is transformed and results finally in a picture that shows the sample surface.

\section{Approach}

The first step of the approach, is to model the AFM with the preprocessor GiD (including geometry, meshing, assign materials and boundary conditions).

The physical process is idealised by partial differential equations, whereas the coupling of the mechanical and the electrostatical field is performed by the Maxwell stress tensor. To solve the partial differential equations, the Finite Element Method (FEM) is used (Reddy, 1993). In this context, the elements definitions and the solving of the system of equations are programmed in Matlab. In former studies the electrostatic and mechanical equations were considered to be weak coupled, which means that the mechanical and electrostatical forces are calculated separately (Helmich, 2007; Bala, 2008; Greiff, 2009). In this case convergence of the

Published by Copernicus Publications on behalf of the URSI Landesausschuss in der Bundesrepublik Deutschland e.V. 


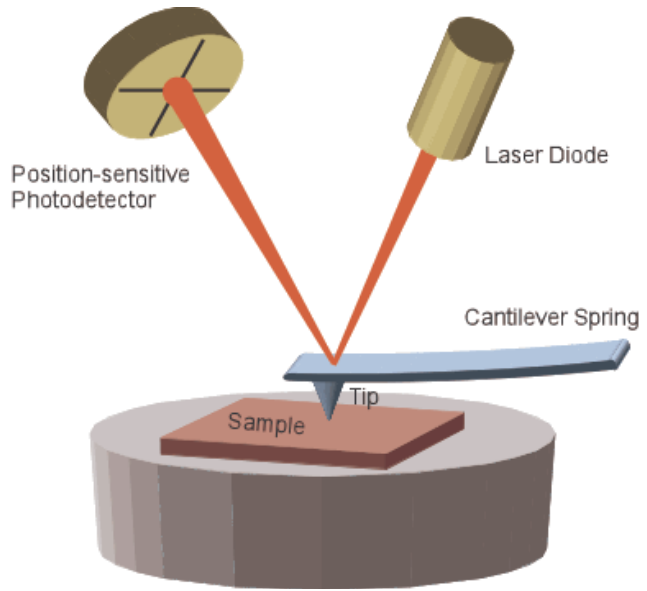

Fig. 1. Principle of an atomic force microscope (Atomic Force Microscope, 2009).

solution is not guaranteed. In this contribution, the partial differential equations are solved by a strong coupled formulation (Fig. 2). If the linearisation of the nonlinear differential equations is consistent, the starting values of the degrees of freedom are near to the solution values and the convergence is guaranteed. At last the postprocessing is done with GiD.

\section{Computational methods}

The equation describing the electrostatical part (Kuepfmueller, 2006) is given by the reduced form of the Maxwell equation

$\operatorname{div}(\boldsymbol{D})=0$,

because of the non-existence of free charges. $\boldsymbol{D}$ denotes the electric flux density. The constitutive equations are given by

$\boldsymbol{D}=\epsilon_{0} \boldsymbol{E}$

and

$\boldsymbol{E}=-\operatorname{grad}(\varphi)$

with the electrical permittivity $\epsilon_{0}$, the electrostatic field $\boldsymbol{E}$ and the electrostatic potential $\varphi$. The mechanical problem is described by the balance of linear momentum

$\operatorname{div}(\boldsymbol{\sigma})+\rho \boldsymbol{b}=\rho \dot{\boldsymbol{v}}$,

however no inertia forces $\rho \boldsymbol{b}$ or volume forces $\rho \dot{\boldsymbol{v}}$ are considered (Wriggers, 2001). Therefore the strong formulation of the balance of linear monumentum is written as

$\operatorname{div}(\boldsymbol{\sigma})=\mathbf{0}$.

$\sigma$ denotes a stress tensor that is given by

$\sigma=\sigma^{M}+\sigma^{C}$,

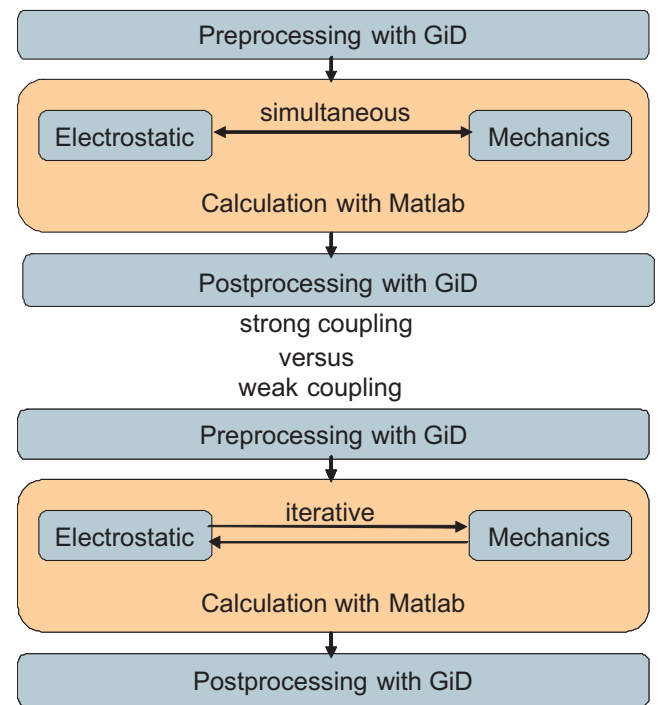

Fig. 2. Process of strong coupling versus weak coupling.

with the Cauchy stress tensor $\sigma^{C}$ and the Maxwell stress tensor $\sigma^{M}$. The Cauchy stress tensor is computed by a linear relation to strains, which is referred to as Hooke's law

$\sigma^{C}=\mathbb{C} \epsilon$.

$\mathbb{C}$ denotes the elastic constitutive tensor. The strain tensor

$\boldsymbol{\epsilon}=\operatorname{grad}^{s}(\boldsymbol{u})=\frac{1}{2}\left(\operatorname{grad}(\boldsymbol{u})+\operatorname{grad}^{t}(\boldsymbol{u})\right)$,

is assumed by the symmetrical part of the displacement gradient. The Maxwell stress tensor $\boldsymbol{\sigma}^{\boldsymbol{M}}$ is given by

$\sigma_{i j}^{M}=\epsilon_{0}\left(E_{i} E_{j}-\frac{1}{2} E_{l} E_{l} \delta_{i j}\right)$.

$\delta_{i j}$ denotes the Kroneker delta with

$\delta_{i j}=1$, for $i=j$

$\delta_{i j}=0$, for $i \neq j$.

\subsection{Variational formulations}

In the following, the weak forms of the mechanical and the electrostatical part of the system are presented. For the mechanical part the strong form (5) is multiplied with the test function $\eta_{\boldsymbol{u}}$, and then integrated over the calculation domain $\Omega$

$\int_{\Omega} \operatorname{div}\left(\sigma^{C}\right) \cdot \eta_{u} d \Omega=0$

Using partial integration and the Gauss' theorem we get

$\int_{\Gamma}\left(\boldsymbol{\sigma}^{C} \boldsymbol{n}\right) \cdot \boldsymbol{\eta}_{\boldsymbol{u}} d \Gamma-\int_{\Omega} \boldsymbol{\sigma} \operatorname{grad}^{s}\left(\boldsymbol{\eta}_{\boldsymbol{u}}\right) d \Omega=0$, 
whereas on the boundary $\Gamma$ only the Maxwell stress tensor and in the region $\Omega$ only the Cauchy stress tensor acts,

$\int_{\Gamma}\left(\boldsymbol{\sigma}^{M} \boldsymbol{n}\right) \cdot \boldsymbol{\eta}_{\boldsymbol{u}} d \Gamma-\int_{\Omega} \boldsymbol{\sigma} \operatorname{grad}^{S}\left(\boldsymbol{\eta}_{\boldsymbol{u}}\right) d \Omega=0$.

Substituting Eqs. (7) and (8), the result for the weak form of the mechanical problem is given by

$$
R_{\mathrm{u}}=\int_{\Gamma}\left(\boldsymbol{\sigma}^{\boldsymbol{M}} \boldsymbol{n}\right) \cdot \boldsymbol{\eta}_{\boldsymbol{u}} d \Gamma-\int_{\Omega} \operatorname{grad}^{S}(\boldsymbol{u}) \mathbb{C} \operatorname{grad}^{S}\left(\boldsymbol{\eta}_{\boldsymbol{u}}\right) d \Omega=0 .
$$

The strong form of the electrostatical part combines Eqs. (1), (2) and (3) to

$\operatorname{div}\left(-\epsilon_{0} \operatorname{grad}(\varphi)\right)=0$.

Equivalent to the derivation of the mechanical weak form, the electrostatical weak form is described by

$R_{\varphi}=\int_{\Omega} \operatorname{grad}(\varphi) \epsilon_{0} \operatorname{grad}\left(\eta_{\varphi}\right) d \Omega=0$.

\subsection{Linearisation}

The degrees of freedom of the coupled 3-D problem are firstly the three components of the displacement vector $u_{x}$, $u_{y}$ and $u_{z}$ and secondly the potential $\varphi$. Since the weak form of the balance of linear momentum depends nonlinearly on the E-field, a direct solution of the multiphysical problem is not possible. This is justified by the use of the Maxwell stress tensor. Thus, the system is solved iteratively by the Newton algorithm. This leads to the coupled system of equations

$\left[\begin{array}{cc}\frac{\partial R_{\mathrm{u}}}{\partial \boldsymbol{u}} & \frac{d R_{\mathrm{u}}}{d \varphi} \\ \frac{\partial R_{\varphi}}{\partial \boldsymbol{u}} & \frac{d R_{\varphi}}{d \varphi}\end{array}\right]\left[\begin{array}{c}\Delta \boldsymbol{u} \\ \Delta \varphi\end{array}\right]=\left[\begin{array}{c}-R_{\mathrm{u}} \\ -R_{\varphi}\end{array}\right]$

The derivatives of $R_{\varphi}$ and $R_{\mathrm{u}}$ can be written as follows:

$\frac{\partial R_{\mathrm{u}}}{\partial \boldsymbol{u}} \cdot \Delta \boldsymbol{u} \widehat{=}-\int_{\Omega} \operatorname{grad}^{S}(\Delta \boldsymbol{u}) \mathbb{C} \operatorname{grad}^{S}\left(\eta_{\boldsymbol{u}}\right) d \Omega$,

$\frac{d R_{\varphi}}{d \varphi} \Delta \varphi \widehat{=} \int_{\Omega} \operatorname{grad}(\Delta \varphi) \epsilon_{0} \operatorname{grad}\left(\eta_{\varphi}\right) d \Omega$

and

$\frac{d R_{\mathrm{u}}}{d \varphi} \Delta \varphi \widehat{=} \int_{\Gamma}\left(\left\{\frac{d \boldsymbol{\sigma}^{M}}{d \varphi} \Delta \varphi\right\} \boldsymbol{n}\right) \cdot \boldsymbol{\eta}_{\boldsymbol{u}} d \Gamma$

As a first approach in this contribution we define that the electrostatic field is independent of the idealised displacement variable. This leads to

$\frac{\partial R_{\varphi}}{\partial \boldsymbol{u}}=\mathbf{0}$
The results of the derivatives, that are included in the stiffness matrix in Eq. (17) are described here in a simplified form. While the term on the left side is present in discretized form, the term on the right side is determined in an analytical form. Equation (20) is solved by the derivative of the Maxwell stress tensor with respect to the electrostatic potential $\varphi$. Using the chain rule we get

$\frac{d \sigma_{i j}^{M}}{d \varphi} \Delta \varphi \widehat{=} \underbrace{\frac{\partial \sigma_{i j}^{M}}{\partial E_{k}}}_{A_{i j k}} \operatorname{grad}(\Delta \varphi)_{k}$,

where $A_{i j k}$ is a third order tensor, that can be solved by partial derivation of $\sigma_{i j}^{M}$ with respect to $E_{k}$. The results of $A_{i j k}$ are

$A_{i j 1}=\left[\begin{array}{ccc}E_{x} & E_{y} & E_{z} \\ E_{y} & -E_{x} & 0 \\ E_{z} & 0 & -E_{x}\end{array}\right]$,

$A_{i j 2}=\left[\begin{array}{ccc}-E_{y} & E_{x} & 0 \\ E_{x} & E_{y} & E_{z} \\ 0 & E_{z} & -E_{y}\end{array}\right]$

and

$A_{i j 3}=\left[\begin{array}{ccc}-E_{z} & 0 & E_{x} \\ 0 & -E_{z} & E_{y} \\ E_{x} & E_{y} & E_{z}\end{array}\right]$.

With implementation of the linear shape functions, the concept of isoparametric elements and Gaussian quadrature, the system now can be solve.

\section{AFM-modell}

For this computation a cantilever with a length of $115 \mu \mathrm{m}$ and height and width of $1 \mu \mathrm{m}$ was modelled. The length of the conical tip is $0.5 \mu \mathrm{m}$. The young's modulus $\mathrm{E}$ is $300 \mathrm{GPa}$ (silicon nitride $\mathrm{Si}_{3} \mathrm{~N}_{4}$ ) and the Poisson ratio $v$ is set to 0.24 (Rombach, 2009). For the finite element mesh, tetrahedral elements where used with overall 3245 nodes and 147575 elements. On the left hand side, the cantilever is fixed in all directions. In this area, a potential of $10 \mathrm{~V}$ is given and on the bottom (to model a sample) a potential of $0 \mathrm{~V}$. In the first step of this project, a sample with a flat surface is selected for the verification of the formulations. The coupling is computed on the boundaries of the cantilever.

\section{Computational results}

Figure 4 shows the potential distribution in a slice through the three-dimensional calculation model. Here, the increased 
$\dot{4}$

Fig. 3. Displacement of an AFM in $[\mu \mathrm{m}]$.

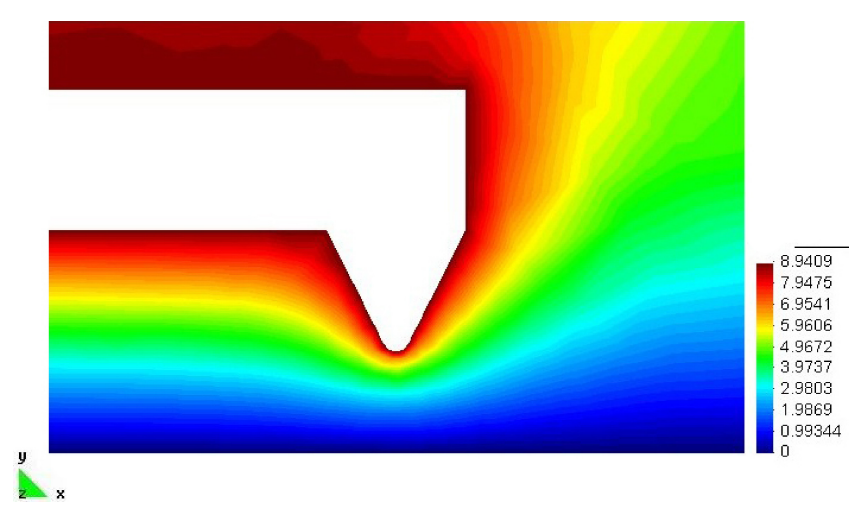

Fig. 4. Potential distribution of an AFM in [V].

gradient is clearly visible at the tip of the cantilever. This is shown significantly by the representation of the electrostatic field in Fig. 5. The combination of the mechanical loads and the electrostatic field by using the Maxwell stress tensor leads to relatively large forces, that act on the tip of the cantilever. The resulting deflection of the cantilever is shown in Fig. 3. On the other boundaries that link the Cantilever with the surrounding medium, the electrostatic field tends against the value 0 , and thus the mechanical loads become negligible. To minimize the computing time, it can be considered to restrict the calculation field to an area around the tip, since mechanical loads are negligible outside this area.

\section{Conclusions}

In this contribution the electromechanical coupling of a working AFM was solved by using FEM. As a first approach, the electrostatic field was adopted as independent of the displacement. Therefore, it is necessary to extend this procedure. Furthermore, the presented results of strong coupling should be compared with those of weak coupling.

Acknowledgements. This work is funded by German Research Foundation (DFG) via a Ph.D. scholarship in the Research Training Group 615.

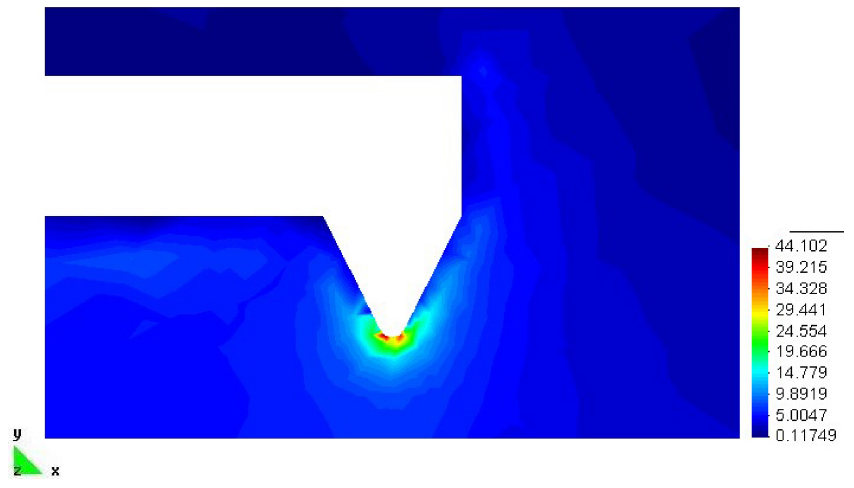

Fig. 5. Electrostatic field of an AFM in [V/ $\mu \mathrm{m}]$.

\section{References}

Atomic Force Microscope: http://www3.physik.uni-greifswald.de/ method/afm/eafm.htm, 2009.

Bala, U. B.: Hybrid Numerical Modelling and Simulation of Electrostatic Force Microscope, Ph.D. thesis, Leibniz Universitaet Hannover, 2008.

Greiff, M.: Ein Konzept zur Modellierung und Simulation mikroelektronischer Systeme mit Anwendung auf ein elektrostatisches Kraftmikroskop, Ph.D. thesis, Leibniz Universitaet Hannover, 2009.

Helmich, T.: Elektromechanisch gekoppelte Kontaktmodellierung auf Mikroebene, Ph.D. thesis, Leibniz Universitaet Hannover, 2007.

Kuepfmueller, K. and Mathis, W. R. A.: Theoretische Elektrotechnik - Eine Einfuehrung, Springer, 17th edn., 2006.

Reddy, J. N.: An Introduction to the Finite Element Method, 2nd edn., 1993.

Rombach, M. and Hollstein, T.: Untersuchungen zum mechanischen Verhalten von Siliciumnitrid in einem Kugel-PlatteKontakt, Materialwissenschaft und Werkstofftechnik, 26, 276282, 2009.

Wriggers, P.: Nichtlineare Finite-Element-Methoden, Springer, 2001. 